\title{
Bulk and Epitaxial $\mathrm{Co}_{2} \mathrm{MnSi}$ Systems with Antisite Disorder: Ab Initio Calculations
}

\author{
K. Carva And I. Turek \\ Charles University, Faculty of Mathematics and Physics \\ Department of Condensed Matter Physics \\ Ke Karlovu 5, 12116 Prague 2, Czech Republic
}

\begin{abstract}
The intermetallic compound $\mathrm{Co}_{2} \mathrm{MnSi}$ is halfmetallic, but the structure of real samples is often affected by antisite disorder. The influence of disorder on transport properties is examined by $a b$ initio calculations and is found to be more significant in thin $\mathrm{Co}_{2} \mathrm{MnSi}$ slabs sandwiched by metallic leads than in the bulk compound.
\end{abstract}

PACS numbers: 72.25.--b, 72.10.Fk

\section{Introduction}

The intermetallic compound $\mathrm{Co}_{2} \mathrm{MnSi}$ is a representative of the wide class of full Heusler alloys. It was found to be halfmetallic by ab initio calculations [1]. One of the most important advantages of $\mathrm{Co}_{2} \mathrm{MnSi}$ over other halfmetals is its high Curie temperature $985 \mathrm{~K}$ and the expected width of the minority band gap. The presence and size of this gap was finally confirmed experimentally by tunneling conductance spectroscopy very recently [2]. However, measured spin polarization is commonly much lower than expected (about 50\%), probably because swapping between Mn atoms and close similar sized Co atoms is likely to occur. The used methods are also often surface sensitive, but the halfmetallic character is destroyed at surfaces. Here we examine the role of disorder in both bulk and epitaxial $\mathrm{Co}_{2} \mathrm{MnSi}$ by means of ab initio calculation methods described in Refs. [3-5].

\section{Bulk properties}

Transport calculations show zero conductivity in the minority spin channel of bulk $\mathrm{Co}_{2} \mathrm{MnSi}$ as expected because of its predicted halfmetallic character (see 


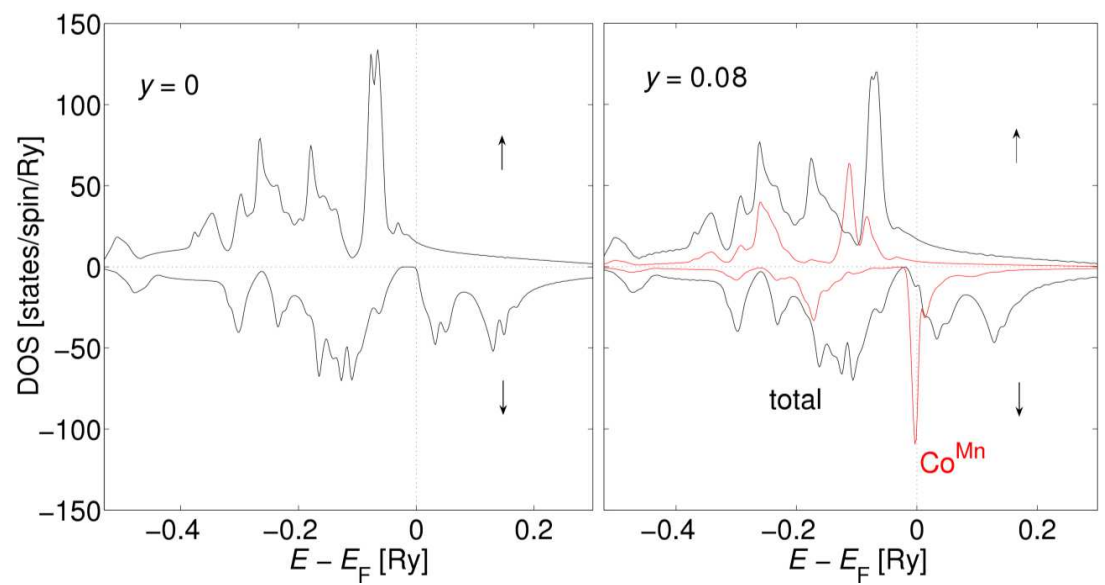

Fig. 1. Density of states of ideal ( $y=0$, left part) and disordered ( $y=0.08$, right part) bulk $\left(\mathrm{Co}_{1-y / 2} \mathrm{Mn}_{y / 2}\right)_{2}\left(\mathrm{Mn}_{1-y} \mathrm{Co}_{y}\right) \mathrm{Si}$.

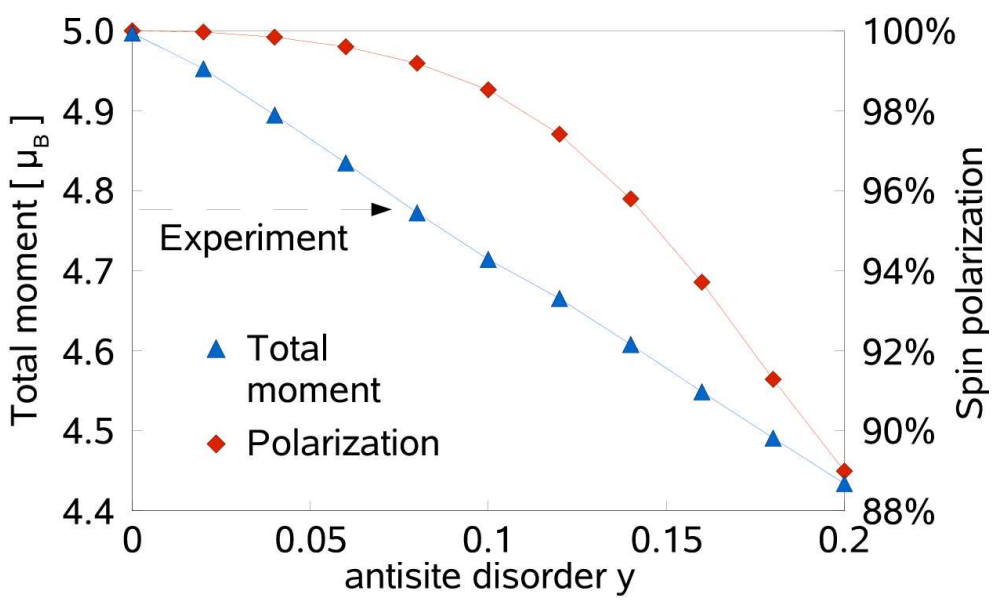

Fig. 2. Total Mn moment and conductivity spin polarization $P$ in disordered bulk $\left(\mathrm{Co}_{1-y / 2} \mathrm{Mn}_{y / 2}\right)_{2}\left(\mathrm{Mn}_{1-y} \mathrm{Co}_{y} \mathrm{Si}\right.$ as functions of antisite disorder $y$.

Fig. 1, left part for density of states). Calculated magnetic moment is $5 \mu_{\mathrm{B}}$ per f.u. (formula unit), in agreement with the fact that there must be an integer number of electrons per f.u. present in both spin directions.

SQUID based measurements of samples without special disorder reduction have yielded a value of $4.78 \mu_{\mathrm{B}}$ [6], which may be explained by the presence of antisite disorder. Alloys $\left(\mathrm{Co}_{1-y / 2} \mathrm{Mn}_{y / 2}\right)_{2}\left(\mathrm{Mn}_{1-y} \mathrm{Co}_{y}\right) \mathrm{Si}$ with swapping between Co and $\mathrm{Mn}$ in the range of up to $y=20 \%$ Co as antisites on the Mn sublattice has been investigated (Fig. 2) employing the coherent potential approximation 
(CPA) for bulk transport [4]. The corresponding range of calculated magnetic moments comprises also the experimental value of $4.78 \mu_{\mathrm{B}}$, which can be matched to $y=8 \%$. X-ray measurements [7] (on different samples) have shown a similar value of $y=7 \%$, which corroborates that disorder of these non-negligible values can be generally expected in samples without a special treatment. This amount of disorder corresponds to conductivity spin polarization $P=99.2 \%$ (Fig. 2), where $P=\left(C_{\uparrow}-C_{\downarrow}\right) /\left(C_{\uparrow}+C_{\downarrow}\right)$ and $C_{\uparrow, \downarrow}$ denote majority and minority conductivity (or conductance in the next section). The calculated density of states shows that a Co impurity band is formed in the minority channel close to the Fermi energy (Fig. 1, right part). Halfmetallicity is destroyed, but the conductivity due to such a virtual bound state remains strongly suppressed. Hence the conductivity spin polarization is still high, but the non-negligible number of states near $E_{\mathrm{F}}$ in the minority channel may significantly impact spin polarization measurements.

\section{Thin layers}

Thin layers of $\mathrm{Co}_{2} \mathrm{MnSi}$ were examined as sandwiched by bcc $\mathrm{Cr}(001)$ leads as motivated by recent experiments [2], see [8] for more details about the used model and computational method. The slabs comprised an even number of atomic layers $n$, where $4 \leq n \leq 26$, they were thus terminated by a Co atomic layer on one side of the junction and by a layer containing $\mathrm{Mn}, \mathrm{Si}$ on the other side. For ideal slabs the minority current-perpendicular-to-plane (CPP) conductance is suppressed very quickly (Fig. 4a of Ref. [8]), which obviously leads to a quick saturation of conductance spin polarization.

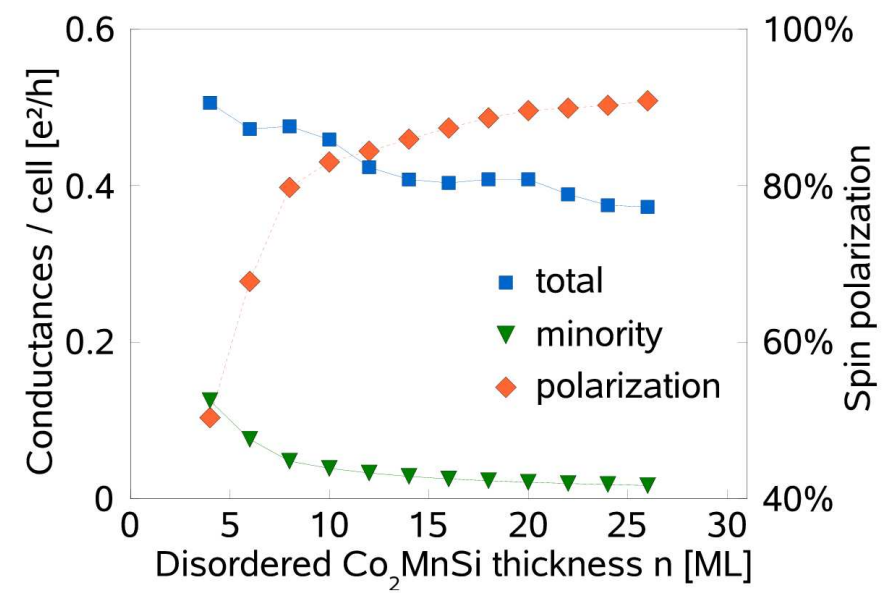

Fig. 3. CPP conductances $C, C^{\downarrow}$ and conductance spin polarization $P$ of $\left(\mathrm{Co}_{0.96} \mathrm{Mn}_{0.04}\right)_{2}\left(\mathrm{Mn}_{0.92} \mathrm{Co}_{0.08}\right) \mathrm{Si}$ (001) slabs sandwiched by two semi-infinite nonmagnetic bcc $\mathrm{Cr}(001)$ leads as functions of its thickness $n$. 
The CPP conductances for samples with the expected amount of disorder $(y=8 \%)$ were calculated (Fig. 3) using the recently introduced CPA based calculation scheme for transport in disordered layered systems [5]. Let us note that while for the bulk case this amount of disorder led to relatively very small reduction of the spin polarization $(100 \% \rightarrow 99.2 \%$, see Fig. 2$)$, the CPP conductance polarization of the thin layer remains at significantly lower values even for the thickness of 24 monolayers. This thickness was enough to yield $99.99 \%$ polarization for pure thin layers, but for $\left(\mathrm{Co}_{0.96} \mathrm{Mn}_{0.04}\right)_{2}\left(\mathrm{Mn}_{0.92} \mathrm{Co}_{0.08}\right) \mathrm{Si}$ it is reduced to $90.2 \%$. The minority conductance now has apparent ohmic behavior for larger

thickness, it is thus reduced mainly due to strong scattering and not tunneling, as suggested from the analysis of the bulk density of states (DOS).

\section{Acknowledgments}

This work is a part of the research plan MSM 0021620834 that is financed by the Ministry of Education of the Czech Republic.

\section{References}

[1] S. Ishida, T. Masaki, S. Fujii, S. Asano, Physica B 245, 1 (1998).

[2] Y. Sakuraba, M. Hattori, M. Oogane, Y. Ando, H. Kato, A. Sakuma, T. Miyazaki, H. Kubota, Appl. Phys. Lett. 88, 192508 (2006).

[3] I. Turek, V. Drchal, J. Kudrnovský, M. Šob, P. Weinberger, Electronic Structure of Disordered Alloys, Surfaces and Interfaces, Kluwer, Boston 1997.

[4] I. Turek, J. Kudrnovský, V. Drchal, P. Weinberger, J. Phys., Condens. Matter 16, S5607 (2004).

[5] K. Carva, I. Turek, J. Kudrnovský, O. Bengone, Phys. Rev. B 73, 144421 (2006).

[6] L. Ritchie, G. Xiao, Y. Ji, T.Y. Chen, C.L. Chien, M. Zhang, J. Chen, Z. Liu, G. Wu, X.X. Zhang, Phys. Rev. B 68, 104430 (2003).

[7] B. Ravel, M.P. Raphael, V.G. Harris, Q. Huang, Phys. Rev. B 65, 184431 (2001).

[8] I. Turek, K. Carva, J. Phys., Condens. Matter 19, 365203 (2007). 